\title{
Propiedades Psicométricas del Inventario de Asertividad de Gambrill y Richey en Estudiantes Universitarios Chilenos
}

\author{
Psychometric Properties of Gambrill and Richey's Assertiveness Inventory on \\ Chilean University Students
}

\author{
Gracia Navarro Saldaña ${ }^{1}$, Marcela Varas Contreras $^{2}$ y Jorge Maluenda Albornoz ${ }^{3}$
}

\begin{abstract}
Resumen
La presente investigación se contextualiza en el diseño, implementación y validación de un modelo educativo para formar en Competencias Genéricas de una universidad chilena. Busca obtener medidas de validez y confiabilidad que respalden el uso del Inventario de Asertividad de Gambrill y Richey en dicho contexto. Se realiza un estudio descriptivo sobre validez de constructo, a través de Análisis Factorial Exploratorio con rotación Oblicua (Promax), y sobre confiabilidad, a partir del coeficiente Alfa de Cronbach. La muestra se conforma de 759 estudiantes universitarios, 50,1\% mujeres y $49.9 \%$ hombres, cuyas edades fluctúan entre los 18 y los 46 años, con media de 20,3 y desviación estándar 2,3 años. Se obtiene una estructura de 6 factores, los que explican el $51.45 \%$ y el $50.05 \%$ de la varianza total para la escala Grado de Incomodidad (GI) y Probabilidad de Respuesta (PR) respectivamente. Se obtiene una elevada confiabilidad con $\mathrm{a}=.93$ para $\mathrm{GI}, \mathrm{a}=.92$ para $\mathrm{PR}$ y $\mathrm{a}=.95$ para la escala total.
\end{abstract}

Palabras clave: asertividad, competencias genéricas, validez, confiabilidad

\begin{abstract}
The following research is contextualized in the design, implementation and validation of an educational model to develop Generic Competences in a chilean university. It looks to obtain reliability and validity measures that support the use of the Gambrill and Richey's Assertiveness Inventory in that context. A descriptive study is made for construct validity, through an Exploratory Factor Analysis with Oblique rotation (Promax), and on reliability through the Alfa Cronbach coefficient. The sample is made up of 759 university students, $50.1 \%$ female and $49.9 \%$ male, with ages fluctuating between 18 and 46 years, with a mean of 20.3 and standard desviation of 2.3 years. The result is a 6 factor structure, that explain 51,45\% and $50.05 \%$ of the total variation for Discomfort (D) and Response Probability (RP) respectively A high reliability is obtained, with $\mathrm{a}=.93$ for $\mathrm{D}, \mathrm{a}=.92$ for $\mathrm{RP}$ and $\mathrm{a}=.95$ for the total scale.
\end{abstract}

Keywords: assertiveness, generic competences, validity, reliability

\footnotetext{
1 Psicóloga, Doctora en Educación y Magister en Responsabilidad Social. Profesora asociada del Departamento Psicología de la Universidad de Concepción. Dirección postal: Víctor Lamas 1290, Concepción, Chile. 56412203037. Correo: gnavarro@udec.cl

2 Ingeniero Civil Informático. Jefe de la Unidad de Investigación y Desarrollo Docente de la Universidad de Concepción. Dirección postal: Víctor Lamas 1290, Concepción, Chile. 56 412203037. Correo: mvaras@udec.cl

${ }^{3}$ Psicólogo y Magister en Política y Gobierno. Asesor en Competencias Genéricas de la Universidad de Concepción. Víctor Lamas 1290, Concepción, Chile. 56 412203037. Correo: jorgemaluendaa@gmail.com
} 


\section{Introducción}

\section{Asertividad y la formación de profesionales}

La competencia profesional es entendida como un complejo sistema de conocimiento, creencias y acciones construidos a partir del dominio bien organizado de distintos saberes (Simone, 2002) donde adquiere importancia el buen desempeño en contextos diversos y auténticos, basado en la integración y activación de los mismos (Villa \& Poblete, 2007). Lo anterior, implica el dominio del alumno sobre conocimientos (saber), habilidad en las técnicas requeridas (hacer) y la capacidad de desenvolverse (ser) (Calderón, 2012).

Es así, que en la actualidad, el "ser competente" profesionalmente, se entiende como un fenómeno complejo, que incluye la expresión de potencialidades, el manejo de conocimientos, habilidades, motivos y valores, orientando las acciones profesionales hacia un desempeño eficiente, ético y con compromiso social (González \& González, 2008).

En este nuevo contexto, las habilidades interpersonales, entre las que se encuentra la asertividad, adquieren un rol indispensable en la formación de profesionales que sean capaces de interactuar con éxito frente a los desafíos laborales y sociales en que se encuentran inmersos.

La asertividad puede ser entendida según Paterson y Rector (2001) como la capacidad de un individuo para transmitir a otra persona sus posturas, opiniones, creencias o sentimientos de manera eficaz, sin sentirse incómodo y respetando los derechos de los demás. A partir de esta conducta, la persona es capaz de asegurar y ratificar las opiniones propias en situaciones interpersonales, manteniendo autorefuerzos sin utilizar conductas agresivas hacia los otros (Casas-Anguera et al., 2014).

Estas características contribuirían al establecimiento y desarrollo de relaciones efectivas, benéficas y satisfactorias con los demás (Paterson \& Rector, 2001) donde las habilidades comunicativas, el control de impulsos y el manejo de emociones, adquieren relevancia para el establecimiento de relaciones más satisfactorias (Velásquez, Arellanez, \& Martínez, 2012).
Por el contrario, la falta de asertividad implica dificultades tanto para poner límites a otras personas y diferenciarse de ellas, como para expresar juicios discrepantes de manera independiente, y la expresión de los propios sentimientos y opiniones (Behar, Manzo, \& Casanova, 2006). Todo ello, repercute de manera negativa en las habilidades de la persona para disfrutar de las relaciones y para cumplir sus metas personales pudiendo conducir, de este modo, al desarrollo de psicopatologías y al deterioro social y ocupacional (Castaños, Reyes, Rivera, \& Díaz, 2011).

Distintas investigaciones han observado diversas relaciones entre asertividad y variables vinculadas a la formación de personas. Se ha observado una relación directamente proporcional entre asertividad y autoestima en el contexto educativo (León, Rodríguez, Ferrel, \& Ceballos, 2009), su importancia en el desenvolvimiento escolar (Caso-Niebla \& Hernández-Guzmán, 2007) y el valor de su dimensión afectiva como elemento motivador para la transmisión y apropiación de los conocimientos relacionados a la enfermería (Pardo, Santacruz, Ramos, \& Arango, 2010). Junto a ello, se aprecia la asertividad como competencia del capital humano requerida en puestos de trabajo de los graduados de educación superior en Europa (Vila, 2005). Más aún, ser profesional asertivo otorga beneficios en el área personal, aumentando la autoestima, la seguridad personal, la autoimagen y el autoconcepto, como también en el área laboral, disminuyendo el número de situaciones estresantes e incrementando la eficacia, calidad y competencia de los cuidados y servicios que se prestan (Cañón-Montañez \& Rodríguez-Acelas, 2011); razones que fundamentan la importancia de contar con herramientas adecuadas para medir la asertividad en el contexto de la educación superior, de modo de entregar información precisa a los distintos actores del proceso educativo, que permitan mejorar su desarrollo.

Estas herramientas adquieren mayor relevancia aún desde el enfoque de la Educación Basada en Competencias (EBC) donde las habilidades interpersonales y comunicacionales se ubican en el mismo nivel de importancia que los conocimientos y la técnica, para contribuir a la 
formación de profesionales con capacidad de afrontar los desafíos que la sociedad les presenta.

\section{Medidas de asertividad: Inventario de Asertividad de Gambrill y Richey}

Se han desarrollado diversas escalas que buscan la medición del constructo asertividad. Entre ellas se encuentra la Escala de Asertividad de Wolpe y Lazarus (1966), el Inventario de Asertividad de Gambrill y Richey (1975), la Escala de Autoexpresión para Adultos de Galassi, Delo, Galassi y Bastien, (1974) la Escala de Asertividad de Rathus, (1973) y el Inventario de Resolución de Conflictos de McFall y Lillesand, (1971), entre otros.

Se han observado diversas dificultades entre estas escalas, que afectan su validez y confiabilidad, principalmente, en relación a la operacionalización de la asertividad y la multidimensionalidad de esta (Castaños et al., 2011). En el primer caso, las conductas que representan a la asertividad son distintas para cada contexto sociocultural mientras que, en el segundo, cada instrumento mide diferentes partes de esta (Ibíd.).

El inventario de Asertividad de Gambrill y Richey (1975) abarca una amplia variedad de situaciones interpersonales considerando con ello, la multidimensionalidad del constructo. Además, relaciona un factor de naturaleza emocional (incomodad subjetiva) con su manifestación conductual (Castaños et al., 2011) permitiendo mayor amplitud en la medición.

Por otro lado, ha sido adaptado al español mostrando adecuadas propiedades psicométricas en población hispanohablante, razones que le sitúan como una buena alternativa para la medición de la Asertividad en distintos contextos.

Gambrill y Richey (1975) organizaron este inventario en dos Subescalas, Grado de Incomodidad (GI) y Probabilidad de Respuesta (PR), incluyendo un total de 40 reactivos, en las que se presentan situaciones de interacción con extraños, amigos, compañeros de trabajo y personas íntimamente relacionadas. Agruparon los reactivos en ocho categorías: "Rechazar peticiones", "Admitir limitaciones personales", "Iniciar contactos sociales", "Expresar sentimientos positivos", "Realizar conductas asertivas en lugares públicos", Discrepar de las opiniones de otros", "Afrontar las críticas de los demás" y "Expresar sentimientos negativos".

Carrasco, Clemente, y Llanova, (1989), señalaron también ocho grupos de conductas para este inventario que representan en su conjunto el constructo asertividad y que han sido utilizadas para organizar las dimensiones de este comportamiento y los reactivos que puedan dar cuenta de ella.

Proponen "conductas de oposición, enfrenatamieno y defensa"; de "manifestación de opiniones personales"; "dar y aceptar cumplidos"; "hacer peticiones"; "expresar sentimientos positivos o de afecto"; "expresar el desagrado o enfado"; "iniciar y mantener conversaciones"; y "expresar limitaciones personales".

Agregan además una serie de características que deben satisfacer las conductas para ser consideradas parte de la asertividad. Estas deben manifestarse de forma directa, sin ansiedad excesiva e inapropiada y de una manera no amenazante ni punitiva para las demás personas involucradas en la interacción; deben ser rastreadas en distintas áreas de la vida en las que se encuentre el sujeto (familiar, laboral, amistades, servicios); deben considerar la relación entre la persona que emite la conducta asertiva y su interlocutor (extraños, diferente sexo, figuras de autoridad y edad).

Todos estos aspectos se consideran importantes en tanto que afectan diferencialmente la emisión de la conducta asertiva (Carrasco et al., 1989), por lo tanto, deben ser considerados a la hora de concebir las dimensiones de medición así como los reactivos que contrubyen a estas.

Mas actualmente, (Castaños et al., 2011) han observado siete factores comunes a ambas escalas: "Manejar situaciones de servicio"; "Confrontación"; "Admitir deficiencias personales; "Involucrarse en situaciones agradables"; "Rechazar peticiones"; "Dar retroalimentación negativa"; e "Iniciar interacciones". Además, concluyen en la presencia de un factor diferenciado para GI (Manejar situaciones molestas) y otro para PR (Resistir la presión) si bien ambos apuntan en la misma dirección y se componen de idénticos reactivos. 
El modo de respuesta de este inventario se organiza a través de una escala tipo Likert, el cual, ha mostrado puntuaciones satisfactorias en cuanto a sensibilidad y especificidad (Brabete, 2014). A partir de este método, los participantes deben señalar su grado de incomodidad, y luego, indicar la probabilidad de llevar a cabo la conducta.

La versión original del inventario obtuvo un coeficiente de confiabilidad test-retest de .87 para la subescala GI y de .81 para PR. En esta oportunidad, sólo se realizó el análisis factorial para el indicador GI sin reporte de razones para ello. La estructura factorial obtenida fue coherente con las categorías que engloban los reactivos (Castaños et al. 2011).

Además, para la misma versión, en un estudio compartivo de población esquizofrénica con población normal, se observaron buenas medidas de validez convergente con el Cuestionario de Habilidades de Comunicación (CSQ) y validez divergente con otras escalas de funcionamiento social (Casas-Anguera et al., 2014).

Castaños et al. (2011) realizan la validación de la versión adapatada por Guerra (1996) al contexto mexicano, utilizando una muestra de la población general, desde la que resulta la segunda versión del instrumento. En ella, se eliminó el reactivo 22 "decirle a alguien que le cae muy bien" ya que, según los resultados obtenidos, no aporta a la discriminación del constructo en cuestión, por lo que la escala final se compone de 39 reactivos. Además, Castaños et al. (2011) repotan la reelaboración de los reactivos $3,9,18$, 20 y 36, basados en revisión de literatura, debido a su discordancia con los factores propuestos por Gambrill y Richey (1975).

Los resultados mostraron que estos 39 ítems son eficaces para medir el constructo, demostrando su confiabilidad y validez. Los valores obtenidos para la confiabilidad, medidos a través del índice Alfa de Cronbach alcanzaron, para la escala general, una significancia de $.94, \mathrm{y}$ $\begin{array}{lllllllll}\text { para las subescalas } & \text { GI } & \text { y } & \text { PR } & .91 & \text { y } & .88\end{array}$ respectivamente. Estos resultados fueron superiores a los obtenidos por Guerra (1996), quien obtuvo .89 para GI y .92 para el inventario total, sin reportar valores para PR (Castaños et al., 2011) valores considerablemente altos teniendo en cuenta la baja cantidad de reactivos del instrumento. Adicionalmente, Castaños et al. (2011) obtienen un coeficiente de confiabilidad temporal para GI de $\mathrm{r}=.50$ y para $\mathrm{PR}$ de $\mathrm{r}=.55$ $(p<.01)$, mostrando ser una medida estable en el tiempo.

A partir del análisis factorial de componentes principales con rotación ortogonal (varimax) en ambas subescalas, Castaños et al., (2011) encuentran que los 39 reactivos alcanzan puntajes superiores a .40 , superiores también a los alcanzados en el estudio de Guerra (1996)

Estos autores corroboran la estructura de ocho factores por subescala encontrando que dichos factores explican un $54.68 \%$ en GI y un $52.36 \%$ PR de la varianza total. Además, al analizar la relación entre los factores a partir del coefficiente de correlación producto-momento de Pearson, observaron correlaciones significativas $(\mathrm{p}<.01)$, en su mayoría positivas y moderadas.

Los resultados antes expuestos sugieren buena posibilidad de persistencia en diferentes tipos de población toda vez que, sus propiedades psicométricas se han mantenido adecuadas habiendo sido utilizado en distintos contexto socioculturales y de distinta habla, incluído el español.

Hasta la fecha, se adolece de estudios sobres este inventario en Chile, y por consiguiente, en población universitaria que permitan contar con información que respalde la validez y confiabilidad del instrumento en dicho contexto.

A raíz de ello, la presente investigación se plantea como objetivo obtener medidas de confiabilidad y validez que respalden el uso de este inventario para la medición de la asertividad en población universitaria chilena.

Considerando como respaldo los estudios realizados previamente en relación a este inventario tanto en su versión original como en la versión adaptada para el habla hispana (Gambrill \& Richey, 1975; Carrasco, et. al., 1989; Castaños, et. al., 2011; Casas-Anguera, et. al. 2014), la presente investigación se plantea como objetivo obtener indices de confiabilidad y validez que puedan ser considerados indicadores veraces de las propiedades psicométricas de esta escala de modo de contar con un instrumento confiable, válido y sensible al contexto chileno. 
Basado en la revisión teórica antes realizada y en las investigaciones citadas, la hipótesis de investigación sugiere que el inventario de Gambrill y Richey es un instrumento válido y confiable para la medición de la Asertividad en población universitaria chilena.

\section{Método}

\section{Participantes}

La muestra se conforma de un grupo de 759 estudiantes universitarios, quienes de forma voluntaria participan de la aplicación del instrumento. Estos se distribuyen en un 50,1\% de mujeres y un $49.9 \%$ de hombres cuyas edades fluctúan entre los 18 y los 46 años, con una media de 20,3 años y desviación estándar de 2,3 años. Cursan carreras pertenecientes a las áreas FísicoMatemática (30,9\%), Social-Humanista (14,8\%) y Químico-Biológica (54,3\%).

\section{Instrumento}

El Inventario de Asertividad de Gambrill y Richey está conformado por dos Subescalas, Grado de Incomodidad (GI) y Probabilidad de Respuesta (PR) e incluye un total de 40 reactivos que plantean situaciones de interacción con extraños, amigos, compañeros de trabajo $y$ personas íntimamente relacionadas.

Los reactivos apuntan a indagar sobre 8 categorías específicamente:

1. Rechazar peticiones.

2. Admitir limitaciones personales.

3. Iniciar contactos sociales.

4. Expresar sentimientos positivos.

5. Realizar conductas asertivas en lugares públicos.

6. Discrepar de las opiniones de otros.

7. Afrontar las críticas de los demás.

8. Expresar sentimientos negativos.

El modo de respuesta se organiza a través de una escala tipo likert desde 1 ("nada") a 5 ("demasiado"), para señalar el grado de incomodidad o malestar subjetivo que experimentaría en distintas situaciones que apuntan a las categorías descritas, y luego, en una escala de 1 ("siempre lo haría") a 5 ("nunca lo haría"), indicar la probabilidad de que lleve a cabo dicha conducta. Puntajes altos en cualquiera de estas escalas indican un menor grado de Asertividad y viceversa.

\section{Diseño y procedimiento}

Se realiza un estudio descriptivo (Hernández, Fernández, \& Baptista, 2010) en tanto busca la descripción de las características del problema en cuestión, en este caso, las características o propiedades psicométricas del instrumento.

Se solicita a los jefes de carrera de cada facultad de la universidad informar a los estudiantes sobre el estudio en cuestión para pedir su participación voluntaria en este. Se informa a estos sobre los objetivos del estudio y las características de la tarea que deben realizar.

Los estudiantes reciben el inventario de manera electrónica a través de una plataforma para efectuar "survey". A través de este mismo método, responden acorde al procedimiento estipulado por los autores. Este procedimiento se encuentra explicado apropiadamente a través de la plataforma que soporta el inventario. $\mathrm{La}$ recopilación de los datos se efectúa también de manera electrónica a partir de la misma plataforma.

Se utiliza el paquete estadístico SPSS para el análisis estadístico, abordando la Validez de Constructo de ambas escalas a partir del Análisis Factorial Exploratorio (AFE) por el método de Componentes Principales con Rotación Oblicua (Promax). Previamente, se comprueba la pertinencia del análisis a partir de la medida de adecuación muestral Kaiser Meyer Olkin (KMO) y la prueba de esfericidad de Bartlett. Todos estos análisis son realizados por subescala.

El análisis de la Confiabilidad del inventario se realiza a partir del Análisis de Consistencia Interna mediante el Coeficiente Alfa de Cronbach para ambas escalas, y luego, para la escala total.

\section{Resultados}

Previo al AFE se realiza la prueba KaiserMeyer-Olkin (KMO) para ambas escalas obteniendo 949 para la escala Grado de incomodidad y .927 para la escala Probabilidad de Respuesta. Del mismo modo se procede a realizar la Prueba de esfericidad de Bartlett encontrando 
resultados estadísticamente significativos para ambas escalas.

Tabla 1. KMO y prueba de Bartlett

\begin{tabular}{lc}
\hline \multicolumn{2}{c}{ Escala Grado de Incomodidad } \\
\hline $\begin{array}{l}\text { Medida de adecuación } \\
\text { muestral de Kaiser-Meyer- }\end{array}$ \\
$\begin{array}{l}\text { Olkin. } \\
\text { Prueba de Esfericidad de } \\
\text { Bartlett }\end{array}$ \\
\hline \multicolumn{2}{c}{ Escala Grado de Incomodidad } \\
\hline $\begin{array}{l}\text { Medida de adecuación } \\
\text { muestral de Kaiser-Meyer- }\end{array}$ \\
$\begin{array}{l}\text { Olkin. } \\
\text { Prueba de Esfericidad de } \\
\text { Bartlett }\end{array}$ \\
\hline
\end{tabular}

Para dar respuesta a la primera hipótesis, se realiza un Análisis Factorial Exploratorio (AFE) con Análisis de Componentes Principales y rotación Oblicua (Promax). Se obtiene una estructura factorial compuesta por seis factores (Ver Tabla 2) por cada subescala que en conjunto explican el $51.458 \%$ y el $50.053 \%$ de la varianza total para GI y para PR respectivamente (Tablas 2 y 3). Tanto los datos porcentuales sobre la varianza explicada como distintos gráficos de sedimentación obtenidos indican el poco aporte de la incorporación de más factores en cada subescala $(2 \%)$.

Gráfico 1. Gráfico de Sedimentación Escala Grado de Incomodidad

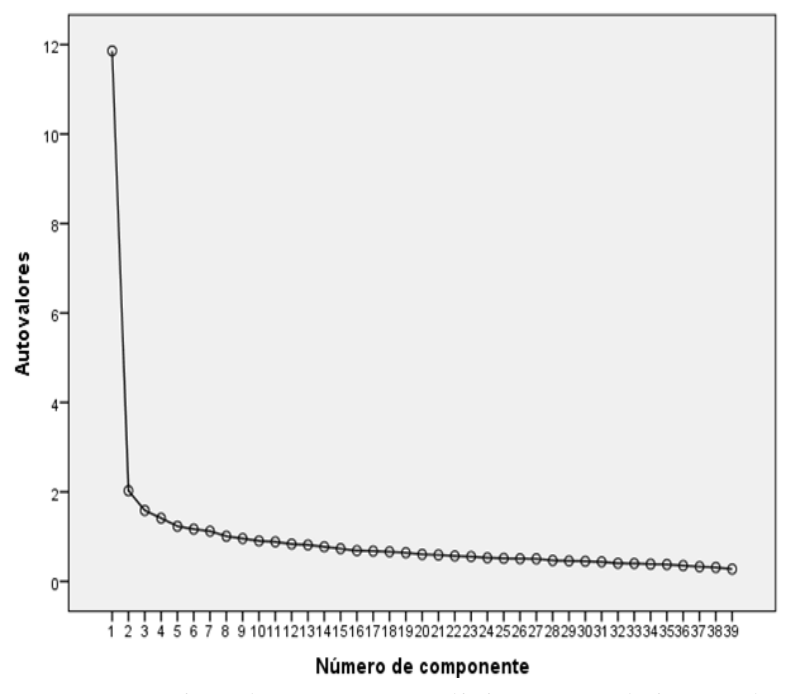

A partir de este análisis se obtiene la estructura factorial del instrumento así como el comportamiento de cada reactivo en relación a cada subescala lo que permite definir los factores relevantes y la organización de los reactivos en
Gráfico 2. Gráfico de Sedimentación Escala Probabilidad de Respuesta

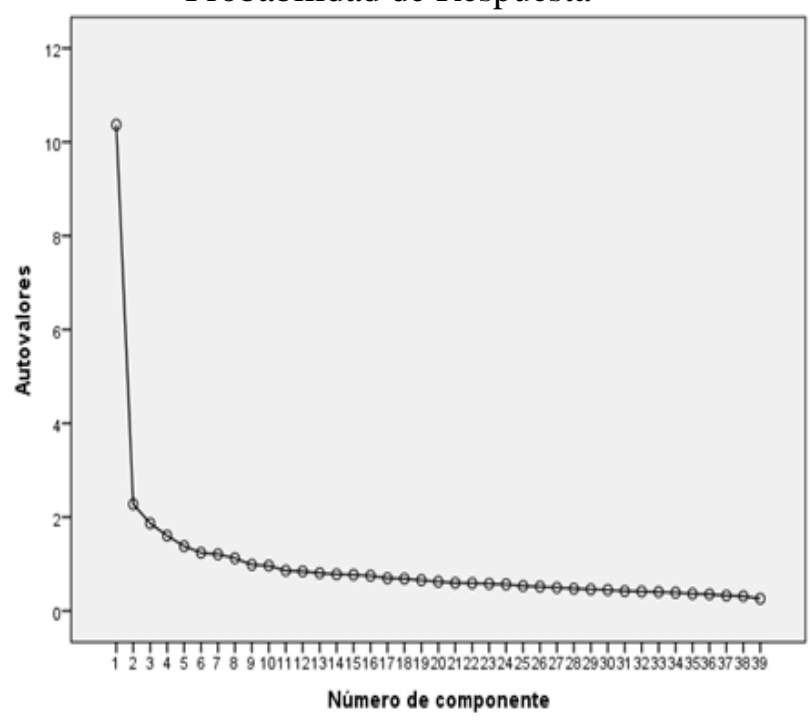

torno a estos factores. El análisis estadístico basado en el AFE realizado se complementa con un análisis teórico basado en la revisión de literatura realizada y los resultados obtenidos en estudios precedentes para habla hispana (Gambrill \& Richey, 1975; Carrasco, et. al., 1989; Castaños, et. al., 2011; Casas-Anguera, et.al., 2014).

A raíz de ello, se excluye el reactivo 22 del análisis propuesto en concordancia con los resultados obtenidos previamente por Castaños, et. al., (2011). Este es excluído por presentar características de una conducta típica, en tanto que, la mayoría de las personas no tiene dificultad alguna para llevarla a cabo, con un consiguiente bajo aporte a la explicación del constructo. Con esto el inventario final se compone de 39 reactivos en lugar de los 40 originales propuestos por Gambrill y Richey (1975) para cada subescala.

Como es posible apreciar a partir de las Tablas 4 y 5 existe una variación entre las escalas GI y PR en cuanto a la ordenación de los factores siendo estas diferencias producto de una distinta agrupación del peso factorial para ambas. Sin perjuicio de aquello, se mantienen identicos factores con una agrupación de reactivos similar. Se aprecia además una variación en tres reactivos específicos $(4,13,33)$ expresando mayor correlación para unos factores que para otros dependiendo de la escala. 
Tabla 2. Estructura factorial escala Grado de Incomodidad

1

GI1. Decirle a alguien que le pide su automóvil, que no puede prestárselo

GI2. Halagar a un amigo (a)

GI3. Admitir que tiene dificultades y que necesita ayuda

GI4. Resistir la tentación de comprar algo en oferta

GI5. Pedir una disculpa

.751 575

.354

.654

GI6. Rechazar la petición de alguien para tener una reunión o una cita

GI7. Admitir que tiene miedo

GI8. Decirle a una persona, con quien está íntimamente relacionado (a) que a usted le molesta algo que dice o hace

GI9. Llamarle la atención al vendedor de la tienda cuando usted ha estado esperando que lo atiendan y atiende primero a una persona que llegó después que usted.

GI10. Aceptar que no sabe sobre algún tema

GI11. Negarse a prestar dinero

GI12. Hacer preguntas personales

G13. Cortar la conversación con una amigo que habla mucho

G14. Pedir una crítica constructiva

G15. Iniciar una conversación con un extraño

G16. Halagar a una persona con la cual está usted involucrado(a)

sentimentalmente o interesado(a) en él o ella

G17. Solicitar una reunión o cita

G18. Discutir abiertamente con una persona a la que respeta, cuando no está de acuerdo con su punto de vista

G19. Admitir confusión acerca de un punto en discusión y solicitar aclaración

G20. Acudir a situaciones sociales con nuevas amistades y extraños

G21. Preguntar si usted ha ofendido a alguien

G23. Reclamar un servicio por el cual usted ha esperado sin ser atendido, por ejemplo en un restaurante

G24. Discutir abiertamente con una persona que critica su conducta

G25. Devolver productos defectuosos, por ejemplo en una tienda o restaurante

G26. Expresar una opinión contraria a la de la persona con la que usted está

hablando

G27. Resistir proposiciones sexuales cuando usted no está interesado(a)

G28. Decirle a una persona que siente que ha sido injusta con usted

G29. Aceptar una cita romántica

G30. Contarle a alguien que a usted le ha ido muy bien

G31. Resistir la presión de alguien que insiste en que usted tome bebidas alcohólicas

G32. Decirle a una persona importante para usted que no tiene razón cuando le hace un reclamo injusto

G33. Renunciar a su trabajo o a algo muy importante para usted

G34. Resistir la presión de alguien que quiere hacerlo enojar

G35. Discutir abiertamente con una persona que critica su trabajo

G36. Llamar la atención a una persona a la que usted le ha prestado alguna

cosa, como un libro, y esta persona ha tardado en devolvérsela

G37. Recibir halagos

G38. Continuar la conversación con alguien que está en desacuerdo con usted

G39. Decirle a un compañero(a) de trabajo que algo que dice o hace le molesta a usted

G40. Pedirle a una persona que lo está molestando ante otras personas que deje de hacerlo
.604

.662 
PR1. Decirle a alguien que le pide su automóvil, que no puede prestárselo

$\begin{array}{llccc}2 & 3 & 4 & 5 & 6 \\ & & & 548\end{array}$

PR2. Halagar a un amigo (a)

PR3. Admitir que tiene dificultades y que necesita ayuda

PR4. Resistir la tentación de comprar algo en oferta

PR5. Pedir una disculpa

PR6. Rechazar la petición de alguien para tener una reunión o una cita

PR7. Admitir que tiene miedo y pedir comprensión

PR8. Decirle a una persona, con quien está íntimamente relacionado (a) que a usted le molesta algo que dice o hace

PR9. Llamarle la atención al vendedor de la tienda cuando usted ha estado esperando que lo atiendan y atiende primero a una persona que llegó después que usted.

PR10. Aceptar que no sabe sobre algún tema

PR11. Negarse a prestar dinero

PR12. Hacer preguntas personales

PR13. Cortar la conversación con una amigo que habla mucho

PR14. Pedir una crítica constructiva

PR15. Iniciar una conversación con un extraño

PR16. Halagar a una persona con la cual está usted involucrado(a) sentimentalmente o interesado(a) en él o ella

PR17. Solicitar una reunión o cita

.667

PR18. Discutir abiertamente con una persona a la que respeta, cuando no está de acuerdo con su punto de vista

PR19. Admitir confusión acerca de un punto en discusión y solicitar aclaración

PR20. Acudir a situaciones sociales con nuevas amistades y extraños

PR21. Preguntar si usted ha ofendido a alguien

PR23. Reclamar un servicio por el cual usted ha esperado sin ser atendido, por ejemplo en un restaurante.

PR24. Discutir abiertamente con una persona que critica su conducta

PR25. Devolver productos defectuosos, por ejemplo en una tienda o restaurante

PR26. Expresar una opinión contraria a la de la persona con la que usted está

hablando

PR27. Resistir proposiciones sexuales cuando usted no está interesado(a)

PR28. Decirle a una persona que siente que ha sido injusta con usted

PR30. Contarle a alguien que a usted le ha ido muy bien

PR31. Resistir la presión de alguien que insiste en que usted tome bebidas alcohólicas

PR32. Decirle a una persona importante para usted que no tiene razón cuando le hace un reclamo injusto

PR33. Renunciar a su trabajo o a algo muy importante para usted

PR35. Discutir abiertamente con una persona que critica su trabajo

PR36. Llamar la atención a una persona a la que usted le ha prestado alguna cosa, como un libro, y esta persona ha tardado en devolvérsela

PR37. Recibir halagos

PR38. Continuar la conversación con alguien que está en desacuerdo con usted

PR39. Decirle a un compañero(a) de trabajo que algo que dice o hace le molesta a usted

PR40. Pedirle a una persona que lo está molestando ante otras personas que deje de hacerlo" 
Tabla 4. Factores y distribución de reactivos Subescala Grado de Incomodidad

\begin{tabular}{lc}
\hline \multicolumn{1}{c}{ Factor } & Distribución Probabilidad de Respuesta \\
\hline 1. Manifestar oposición o discrepancia & $8,9,18,23,24,25,26,28,32,35,36,38,39,40$ \\
2. Asumir limitaciones personales & $3,5,7,10,14,19,21$ \\
3. Rechazar solicitudes/peticiones & $1,4,6,11,13$ \\
4. Exponerse a situaciones desconocidas & $15,17,20$ \\
5. Involucrarse en situaciones agradables & $2,12,16,29,30,37$ \\
6. Manejar situaciones incómodas & $27,31,33,34$ \\
\hline
\end{tabular}

Tabla 5. Factores y distribución de reactivos Subescala Probabilidad de Respuesta

\begin{tabular}{lc}
\hline \multicolumn{1}{c}{ Factor } & Distribución Probabilidad de Respuesta \\
\hline 1. Manifestar oposición o discrepancia & $8,9,18,23,24,25,26,28,32,35,36,38,39,40$ \\
2. Asumir limitaciones personales & $3,5,7,10,14,19,21$ \\
3. Exponerse a situaciones desconocidas & $12,15,17,20$ \\
4. Manejar situaciones incómodas & $4,27,31,34$ \\
5. Rechazar solicitudes/peticiones & $1,6,11,13,33$ \\
6. Involucrarse en situaciones agradables & $2,16,29,30,37$ \\
\hline
\end{tabular}

Para dar respuesta a la segunda hipótesis, se realiza el análisis de confiabilidad a partir del coeficiente Alfa de Cronbach por cada subescala. Se obtienen altos valores en relación a Grado de Incomodidad $(\mathrm{a}=.935)$ y Probabilidad de Respuesta $(\mathrm{a}=.921)$ con una confiabilidad para la escala total también alta $(a=.955)$.

\section{Discusión}

Desde las distintas investigaciones observadas en relación a la medición de la asertividad, y especialmente, aquellas referidas al inventario de Gambrill y Richey es posible apreciar puntos de convergencia teórica respecto de la formación en Competencias Genéricas en el nivel universitario.

El inventario, recorre una serie de situaciones que exploran distintas dimensiones del constructo asertividad lo que permite indagar sobre aspectos fundamentales asociados a las Competencias Genéricas que el modelo educativo de la universidad promueve, con un especial énfasis sobre las competencias "Comunicación" y "Trabajo en equipo interdisciplinario y emprendimiento". Su sensibilidad sobre aspectos cognitivos (reconocer y discriminar sobre conductas asertivas y no asertivas) $\mathrm{y}$ afectivos/actitudinales (Manifestar comodidad/agrado o incomodidad/desagrado frente a situaciones, presentar una actitud hacia ciertas conductas) permiten su uso en distintos niveles de formación y con distintos énfasis, siendo una buena medida para determinar diagnósticos y posibles efectos de los programas de intervención orientados a su desarrollo.

Otro aspecto relevante es su capacidad para satisfacer los criterios propuestos por Carrasco, et. al., (1989), considerando el desarrollo de la asertividad con niveles normales de ansiedad, en ausencia de conductas agresivas, desplegadas en distintos contextos y en distintos tipos de relaciones con el interlocutor. Esto puede ser de sumo relevante para la población univseritaria puesto que permiten diferenciar entre aspectos de mayor asertividad respecto de aquellos comunes y realizables por la población en general, agregando sensibilidad al intrumento al realizar las mediciones.

Los aspectos teóricos previamente señalados son respaldados por los resultados obtenidos a partir del AFE y los análisis de consistencia interna del instrumento. Con ello, es posible señalar que desde una perspectiva teórica y estadística el instrumento manifiesta validez de constructo y confiabilidad.

Tanto los resultados obtenidos en cuanto al porcentaje de la varianza explicada como su distribución entre los factores permiten observar coherencia con el modelo teórico a la base sin perjuicio de que, se reduzca en esta investigación 
la cantidad de factores. Estos se diluyen por su baja carga factorial ( $2 \%$ adicional) y por su baja capacidad discriminativa o de aporte a elementos diferenciadores. Por ejemplo, en el caso de los factores "Manejo de situaciones de servicio" y "Dar retroalimentación" presentados por Castaños, et al. (2011) es posible que estos sean explicados por otros factores relacionados y más inclusivos respecto del constructo total; para el primer caso "Manejar situaciones incómodas" y "Exponerse a situaciones desconocidas", en el segundo, "Manifestar oposición o discrepancia".

En cuanto a la agrupación de reactivos por factor se obtienen resultados similares a investigaciones previas, así como se observa similitud en la comparación entre ambas escalas. De hecho, se observa completa identidad entre los reactivos asociados a los factores 1 y 2 , los que explican el porcentaje más amplio de la varianza total, a pesar de la las diferencias en la agrupación entre las subescalas (orden de prioridad de los factores).

Es posible que esto último se deba a la natural diferencia entre la percepción de los sujetos entre sentirse cómodo/incómodo frente a una situación y emprender/no emprender una conducta.

Otro aspecto que merece atención es la translocación entre tres reactivos. Para GI el reactivo número cuatro se agrupa en el factor "Rechazar solicitudes", y el reactivo doce en "Involucrarse en situaciones agradables", mientras que, el reactivo treinta y tres se agrupa en el factor "Manejar situaciones incómodas". Por su parte, en la escala PR el reactivo trinta y tres se agrupa en el factor "Rechazar solicitudes" y el reactivo doce en "Exponerse a siituaciones desconocidas", mientras que, el reactivo cuatro se agrupa en el factor "Manejar situaciones incómodas".

Una posible explicación es aquella basada en la tesis antes expuesta relativa a la distancia natural entre sentirse cómodo/incómodo frente a una situación y emprender/no emprender una conducta. Una explicación alternativa respecto de los reactivos treinta y tres, y cuatro es que, por similitud en su redacción o en el aspecto que intentan medir, no sean lo suficientemente adecuados para discriminar entre los elementos diferenciadores de estos factores.
Así, "Resistir la tentación de comprar algo en oferta" y/o "Renunciar a su trabajo o algo muy importante para Ud." pueden no ser buenos discriminadores entre "Rechazar solicitudes" y "Manejar situaciones incómodas" para esta población.

Estos aspectos sugieren futuras líneas de investigación que permitan ajustar la pertinencia de dichos reactivos en este contexto y en otros que pudiesen ser de interés para el uso del inventario.

A partir de los resultados se concluye la utilidad del instrumento para la medición de la asertividad en población universitaria chilena de acuerdo con las medidas de confiabilidad y validez obtenidas. Una adecuada estructura factorial con coherencia teórica y apropiados niveles de varianza explicada apoyan la validez de constructo de este instrumento. Además, se observa una alta confiabilidad para cada escala y para la escala total, lo que permite pensar en la precisión del instrumento.

En relación a los resultados positivos de esta y otras investigaciones es de esperarse que su transferencia hacia otros contextos tenga buenos resultados sin perjuicio de que deban estudiarse posibles adecuaciones de los reactivos para cada contexto de uso.

\section{Referencias}

Behar, R., Manzo, R., \& Casanova, D. (2006). Trastornos de la conducta alimentaria y asertividad. Revista Médica de Chile, 134, 312-319.

Behar, R., Manzo, R., \& Casanova, D. (2006). Trastornos de la conducta alimentaria y asertividad. Revista Médica de Chile, 134(1), 312-319.

Brabete, A. (2014). El Cuestionario de Salud General de 12 items (GHQ-12): Estudio de traducción y adaptación de la versión. Revista Iberoamericana de Diagnóstico y Evaluación - e Avaliação Psicológica, 1(37), 11-29.

Calderón, M. (2012). Competencias genéricas en enfermeras/os titulados de la Universidad Arturo Pratt, sede victoria, 2010. Ciencia y Enfermería, 18(1), 89-97.

Cañón-Montañez, W., \& Rodríguez-Acelas, A. (2011). Asertividad: Una habilidad social 
necesaria en los profesionales de enfermería y fisioterapia. Texto y Contexto Enfermagem, 20(1), 81-87.

Carrasco, I., Clemente, M., \& Llanova, L. (1989). Análisis del inventario de aserción de Gambrill y Richey. Estudios de Psicología, 37, 63-74.

Casas-Anguera, E., Prat, G., Vilamala, S., Escandell, M., García-Franco, et al., (2014). Validación de la versión española del inventario de asertividad Gambrill y Richey en población con dianóstico de esquizofrenia. Anales de Psicología, 30(2), 431-437.

Caso-Niebla, J., \& Hernández-Guzmán, L. (2007). Variables que inciden en el rendimiento académico de adolescentes mexicanos. Revista Lationamericana de Psicología, 39(3), 487-501.

Castaños, S., Reyes, I., Rivera, S., \& Díaz, R. (2011). Estandarización del Inventario de Asertividad de Gambrill and Richey - II. Revista Iberoamericana de Diagnóstico y Evaluación - e Avaliação Psicológica, 29(1), 27-50.

Galassi, J., Delo, J., Galassi, M., \& Bastien, S. (1974). The college self-expression scale: A measure of assertiveness. Behavior Therapy(5), 165-171.

Gambrill, E., \& Richey, C. (1975). An Assertion Inventory for use in assessment and research. Behavior Therapy, 6, 550-561.

González, V., \& González, R. (2008). Competencias genéricas y formación profesional: Un análisis desde la docencia universitaria. Revista Iberoamericana de Educación(47), 185-209.

Hernández, R., Fernández, C., \& Baptista, P. (2010). Metodología de la investigación. México: Mc Graw Hill.

León, A., Rodríguez, C., Ferrel, F., \& Ceballos, G. (2009). Asertividad y autoestima en estudiantes de primer semestre de la facultad de ciencias de la salud de una univerisdad pública de la ciudad de Santa Marta. Psicología desde el caribe(24), 91-105.

McFall, R., \& Lillesand, D. (1971). Behavioral rehearsal with modeling and coaching in assertion training. Journal of Abnormal Psychology(77), 313-323.
Pardo, M., Santacruz, K., Ramos, Y., \& Arango, C. (2010). Conceptos claves en un programa educativo. Avances en enfermería, 28(especial), 109-119.

Paterson, R., \& Rector, N. (2001). The assertiveness workbook. Canadian Psychology, 42(3), 234-235.

Rathus, S. (1973). A 30-item schedule for assessing assertive behavior. Behavior Therapy(4), 398-406.

Simone, D. (2002). Definition and selection of key competencies. Geneva: Hogrefe y Huber Publisher.

Velásquez, M., Arellanez, J., \& Martínez, A. (2012). Asertividad y consumo de drogas en estudiantes mexicanos. Acta colombiana de psicología, 15(1), 131-141.

Vila, L. (2005). Capital humano heterogéneo y productividad de los graduados de educación superior. Revista económica de Castilla(7), 61-81.

Villa, A., \& Poblete, M. (2007). Aprendizaje basado en competencias: Una propuesta para la evaluación de las competencias genéricas. Bilbao: Ediciones mensajero.

Wolpe, J., \& Lazarus, A. (1966). Behavior Therapy Techniques: A guide to treatment of neuroses. Elmsford, New York: Pergamon press. 